\title{
Compact matrix operators on a new sequence space related to $\ell_{p}$ spaces
}

\section{Abdullah Alotaibi ${ }^{1}$, Mohammad Mursaleen ${ }^{2 *}$ and Syed Abdul Mohiuddine}

\section{"Correspondence:}

mursaleenm@gmail.com

2Department of Mathematics,

Aligarh Muslim University, Aligarh, 202002, India

Full list of author information is available at the end of the article

\begin{abstract}
In this paper, we derive some identities or estimates for the operator norms and the Hausdorff measures of noncompactness of certain matrix operators on the sequence space $\ell_{p}\left(r, s, t ; B^{(m)}\right)$ which is related to $\ell_{p}$ spaces. By applying the Hausdorff measure of noncompactness, we obtain the necessary and sufficient conditions for such operators to be compact. Further, we study some geometric properties of this space.
\end{abstract}

MSC: 46B15; 46B45; 46B50

Keywords: BK space; matrix transformation; Hausdorff measure of noncompactness; compact operator; geometric properties

\section{Background, notation, and preliminaries}

Let $w$ denote the space of all complex sequences $x=\left(x_{k}\right)_{k=0}^{\infty}$. By $\ell_{\infty}, c, c_{0}$, and $\phi$ we denote the sets of all bounded, convergent, null, and finite sequences, respectively. We write $c s$ for the set of all convergent series and

$$
\ell_{p}=\left\{x \in \omega: \sum_{k=0}^{\infty}\left|x_{k}\right|^{p}<\infty\right\} \text { for } 1 \leq p<\infty .
$$

By $e$ and $e^{(n)}(n=0,1, \ldots)$, we denote the sequences with $e_{k}=1$ for all $k$, and $e_{n}^{(n)}=1$ and $e_{k}^{(n)}=0$ for $k \neq n$, respectively. For any sequence $x=\left(x_{k}\right)_{k=0}^{\infty}$, let

$$
x^{[m]}=\sum_{k=0}^{m} x_{k} e^{(k)}
$$

be its $m$-section.

Let $x$ and $y$ be sequences, $X$ and $Y$ be subsets of $\omega$ and $A=\left(a_{n k}\right)_{n, k=0}^{\infty}$ be an infinite matrix of complex numbers. We write $x y=\left(x_{k} y_{k}\right)_{k=0}^{\infty}$,

$$
\begin{aligned}
& Z=x^{-1} * Y=\{x \in \omega: x z \in Y\}, \quad x^{\beta}=x^{-1} * c s, \\
& X^{\beta}=\bigcap_{x \in X} x^{-1} * c s=\left\{a \in \omega: \sum_{k=0}^{\infty} a_{k} x_{k} \text { converges for all } x \in X\right\}
\end{aligned}
$$

for the $\beta$-dual of $X$. Note that $\ell_{\infty}^{\beta}=c^{\beta}=c_{0}^{\beta}=\ell_{1}, \ell_{1}^{\beta}=\ell_{\infty}$ and $\ell_{p}^{\beta}=\ell_{q}$.

\section{Springer}


By $A_{n}=\left(a_{n k}\right)_{k=0}^{\infty}$ and $A^{k}=\left(a_{n k}\right)_{n=0}^{\infty}$ we denote the sequences in the $n$th row and the $k$ th column of $A$, and we write

$$
A_{n}(x)=\sum_{k=0}^{\infty} a_{n k} x_{k} \quad(n=0,1, \ldots)
$$

and $A(x)=\left(A_{n}(x)\right)_{n=0}^{\infty}$, provided $A_{n} \in X^{\beta}$ for all $n$. The set $X_{A}=X(A)=\{z \in \omega: A(z) \in X\}$ is called the matrix domain of $A$ in $X$. Finally $(X, Y)$ denotes the class of all matrices $A$ that map $X$ into $Y$, that is for which $A_{n} \in X^{\beta}$ for all $n$ and $A(x) \in Y$ for all $x \in X$, or equivalently $A \in(X, Y)$ if and only if $X \subset X_{A}$.

The theory of $B K$ spaces is the most powerful tool in the characterization of matrix transformations between sequence spaces.

A sequence space $X$ is called a $B K$ space if it is a Banach space with continuous coordinates $p_{n}: X \rightarrow \mathbb{C}(n \in \mathbb{N})$, where $\mathbb{C}$ denotes the complex field and $p_{n}(x)=x_{n}$ for all $x=\left(x_{k}\right) \in X$ and every $n \in \mathbb{N}$. A $B K$ space $X \supset \phi$ is said to have $A K$ if every sequence $x=\left(x_{k}\right) \in X$ has a unique representation $x=\sum_{k=0}^{\infty} x_{k} e^{(k)}$.

The sequence spaces $\ell_{\infty}, c$, and $c_{0}$ are $B K$ spaces with the same sup-norm given by $\|x\|_{\ell_{\infty}}=\sup _{k}\left|x_{k}\right|$, where the supremum is taken over all $k \in \mathbb{N}$. Further, the space $\ell_{p}$ is a $B K$ space with the usual $\ell_{p}$-norm defined by $\|x\|_{\ell_{p}}=\left(\sum_{k=0}^{\infty}\left|x_{k}\right|^{p}\right)^{1 / p}$, where $1 \leq p<\infty$. Moreover, the $B K$ spaces $c_{0}$ and $\ell_{p}(1 \leq p<\infty)$ have $A K$ [1], Examples 1.13, 1.20.

Let $X$ and $Y$ be Banach spaces. Then $\mathcal{B}(X, Y)$ is the set of all bounded linear operators $L: X \rightarrow Y$, a Banach space with the operator norm defined as usual by

$$
\|L\|=\sup \{\|L(x)\| /\|x\| \leq 1\} \quad(L \in \mathcal{B}(X, Y)) .
$$

If $Y=\mathbb{C}$ then we write $X^{*}$ for the space of all continuous linear functionals on $X$ with the norm defined by

$$
\|f\|=\sup \{|f(x)|:\|x\| \leq 1\} \quad\left(f \in X^{*}\right)
$$

If $X \subset w$ is a normed space and $a \in w$ then we write

$$
\|a\|^{*}=\|a\|_{X}^{*}=\sup \left\{\left|\sum_{k=0}^{\infty} a_{k} x_{k}\right|:\|x\| \leq 1\right\},
$$

provided the expression on the right-hand side exists and is finite which is the case whenever $X \supset \phi$ is a $B K$ space and $a \in X^{\beta}[2]$, p.35.

For any subset $X$ of $w$, the matrix domain of an infinite matrix $A$ in $X$ is defined by

$$
X_{A}=\{x \in w: A x \in X\} .
$$

An infinite matrix $T=\left(t_{n k}\right)$ is called a triangle if $t_{n n} \neq 0$ and $t_{n k}=0$ for all $k>n(n \in \mathbb{N})$. The study of matrix domains of triangles in sequence spaces has a special importance due to the various properties which they have. For example, if $X$ is a $B K$ space then $X_{T}$ is also a $B K$ space with the norm given by $\|x\|_{X_{T}}=\|T x\|_{X}$ for all $x \in X_{T}$ [1], Theorem 4.3.12.

The following known results are fundamental for our investigation [2], Theorem 4.3.12. 


\section{Lemma 1.1}

(a) Let $X$ denote any of the spaces $c_{0}, c, \ell_{\infty}, \ell_{1}$ or $\ell_{p}$. Then we have $\|a\|_{X}^{*}=\|a\|_{X^{\beta}}$ for all $a \in X^{\beta}$, where $\|\cdot\|_{X^{\beta}}$ is the natural norm on the dual space $X^{\beta}$.

(b) Let $X$ and $Y$ be $B K$ spaces. Then every matrix $A \in(X, Y)$ defines an operator $L_{A} \in B(X, Y)$ by $L_{A}(x)=A x$ for all $x \in X$; we denote this by $(X, Y) \subset B(X, Y)$.

(c) Let $X \supset \phi$ be a $B K$ space and $Y$ be any of the spaces $c_{0}, c$ or $\ell_{\infty}$. If $A \in(X, Y)$, then

$$
\left\|L_{A}\right\|=\|A\|_{\left(X, \ell_{\infty}\right)}=\sup _{n}\left\|A_{n}\right\|_{X}^{*}<\infty .
$$

Also, let $\mathcal{F}$ be the collection of all non-empty and finite subsets of $\mathbb{N}=\{0,1,2, \ldots\}$, throughout. Then we have the following result.

Lemma 1.2 Let $X \supset \phi$ be a $B K$ space. If $A \in\left(X, \ell_{1}\right)$, then

$$
\|A\|_{\left(X, \ell_{1}\right)} \leq\left\|L_{A}\right\| \leq 4 \cdot\|A\|_{\left(X, \ell_{1}\right)}
$$

where $\|A\|_{\left(X, \ell_{1}\right)}=\sup _{N \in \mathcal{F}}\left\|\sum_{n \in N} A_{n}\right\|_{X}^{*}<\infty$.

For the reader's convenience, we list a few well-known definitions and results concerning the Hausdorff measure of noncompactness which can be found in [3, 4], and [2].

Let $S$ and $M$ be subsets of a metric space $(X, d)$ and $\varepsilon>0$. Then $S$ is called an $\varepsilon$-net of $M$ in $X$ if for every $x \in M$ there exists $s \in S$ such that $d(x, s)<\varepsilon$. Further, if the set $S$ is finite, then the $\varepsilon$-net $S$ of $M$ is called a finite $\varepsilon$-net of $M$, and we say that $M$ has a finite $\varepsilon$-net in $X$. A subset $M$ of a metric space $X$ is said to be totally bounded if it has a finite $\varepsilon$-net for every $\varepsilon>0$. If $X$ is complete, then $M$ is totally bounded if and only if $M$ is relatively compact (its closure $\bar{M}$ is a compact set). Let $X$ and $Y$ be Banach spaces. A linear operator $L: X \rightarrow Y$ is called compact if $D(L)=X$ for the domain of $L$ and, for every bounded sequence $\left(x_{n}\right)_{n=0}^{\infty}$ in $X$, the sequence $\left(L\left(x_{n}\right)\right)_{n=0}^{\infty}$ has a convergent subsequence in $Y$.

By $\mathcal{M}_{X}$, we denote the collection of all bounded subsets of a metric space $(X, d)$. If $Q \in$ $\mathcal{M}_{X}$, then the Hausdorff measure of noncompactness of $Q$, is defined by

$$
\chi(Q)=\inf \{\varepsilon>0: Q \text { has a finite } \varepsilon \text {-net in } X\} .
$$

The function $\chi: \mathcal{M}_{X} \rightarrow[0, \infty)$ is called the Hausdorff measure of noncompactness.

It is well known that if $Q, Q_{1}$, and $Q_{2}$ are bounded subsets of a metric space $X$, then we have

$$
\begin{aligned}
& \chi(Q)=0 \quad \text { if and only if } Q \text { is totally bounded, } \\
& Q_{1} \subset Q_{2} \quad \text { implies } \quad \chi\left(Q_{1}\right) \leq \chi\left(Q_{2}\right) \\
& \chi(Q)=\chi(\bar{Q}) \quad \text { for the closure } \bar{Q} \text { of } Q \\
& \chi\left(Q_{1} \cup Q_{2}\right)=\max \left(\chi\left(Q_{1}\right), \chi\left(Q_{2}\right)\right) \\
& \chi\left(Q_{1} \cap Q_{2}\right) \leq \min \left(\chi\left(Q_{1}\right), \chi\left(Q_{2}\right)\right) .
\end{aligned}
$$

Further, if $X$ is a normed space then we also have

$$
\chi\left(Q_{1}+Q_{2}\right) \leq \chi\left(Q_{1}\right)+\chi\left(Q_{2}\right)
$$




$$
\chi(\alpha Q)=|\alpha| \chi(Q) \quad \text { for all } \alpha \in \mathbb{C} .
$$

Let $X$ and $Y$ be Banach spaces and $L \in \mathcal{B}(X, Y)$. Then the Hausdorff measure of noncompactness of the operator $L$, denoted by $\|L\|_{\chi}$, is defined by

$$
\|L\|_{\chi}=\chi\left(L\left(S_{X}\right)\right)
$$

where $S=\{x \in X:\|x\| \leq 1\}$ is the unit ball in $X$. Also we have

$$
L \text { is compact if and only if }\|L\|_{\chi}=0 .
$$

Now we shall point out the well-known result of Goldenštein, Gohberg, and Markus [5], Theorem 1, concerning the Hausdorff measure of noncompactness in Banach spaces. The Hausdorff measure of noncompactness of a bounded subset of the $B K$ space $\ell_{p}(1 \leq p<\infty)$ is given by the following result.

Lemma 1.3 Let $X$ be a $B K$ space with $A K$ and monotone norm, $Q \in \mathcal{M}_{X}$, and $P_{n}: X \mapsto X$ $(n \in \mathbb{N})$ be the operator (projection) defined by $P_{n}\left(x_{1}, x_{2}, \ldots\right)=x^{[n]}=\left(x_{1}, x_{2}, \ldots, x_{n}, 0,0, \ldots\right)$ for all $x=\left(x_{1}, x_{2}, \ldots\right) \in X$. Then

$$
\chi(Q)=\lim _{n \rightarrow \infty}\left(\sup _{x \in Q}\left\|\left(I-P_{n}\right) x\right\|\right)
$$

For some recent related work on this topic, we refer to [5-28], and [29]. For some applications in differential and integral equations, we refer to [4, 30-33], and [34].

\section{Results and discussion}

Throughout this paper, let $r, t \in \mathcal{U}$ and $s \in \mathcal{U}_{o}$, where

$$
\mathcal{U}=\left\{u=\left(u_{k}\right) \in w: u_{k} \neq 0 \text { for all } k\right\} \text { and } \mathcal{U}_{o}=\left\{u=\left(u_{k}\right) \in w: u_{0} \neq 0\right\} .
$$

For any sequence $x=\left(x_{n}\right) \in w$, we define the sequence $\bar{x}=\left(\bar{x}_{n}\right)$ of generalized means of $x$ by

$$
\bar{x}_{n}=\frac{1}{r_{n}} \sum_{k=0}^{n} s_{n-k} t_{k} x_{k} \quad(n \in \mathbb{N}) .
$$

Further, we define the infinite matrix $\bar{A}(r, s, t)$ of generalized means by

$$
(\bar{A}(r, s, t))_{n k}= \begin{cases}s_{n-k} t_{k} / r_{n} & (0 \leq k \leq n), \\ 0 & (k>n)\end{cases}
$$

for all $n, k \in \mathbb{N}$. Then, by using the notation of (1.1), it follows by (2.1) that $\bar{x}$ is the $\bar{A}(r, s, t)$ transform of $x$, that is, $\bar{x}=(\bar{A}(r, s, t)) x$ for all $x \in w$. 
Moreover, it is obvious by (2.2) that $\bar{A}(r, s, t)$ is a triangle. Thus, it has a unique inverse $(\bar{A}(r, s, t))^{-1}$ which is also a triangle. More precisely, we put $D_{0}^{(s)}=1 / s_{0}$ and

$$
D_{n}^{(s)}=\frac{1}{s_{0}^{n+1}}\left|\begin{array}{cccccc}
s_{1} & s_{0} & 0 & 0 & \cdots & 0 \\
s_{2} & s_{1} & s_{0} & 0 & \cdots & 0 \\
s_{3} & s_{2} & s_{1} & s_{0} & \cdots & 0 \\
\vdots & \vdots & \vdots & \vdots & & \vdots \\
s_{n-1} & s_{n-2} & s_{n-3} & s_{n-4} & \cdots & s_{0} \\
s_{n} & s_{n-1} & s_{n-2} & s_{n-3} & \cdots & s_{1}
\end{array}\right| \quad(n=1,2,3, \ldots) .
$$

Then the entries of $(\bar{A}(r, s, t))^{-1}$ are given by

$$
(\bar{A}(r, s, t))_{n k}^{-1}= \begin{cases}(-1)^{n-k} D_{n-k}^{(s)} r_{k} / t_{n} & (0 \leq k \leq n) \\ 0 & (k>n)\end{cases}
$$

for all $n, k \in \mathbb{N}$, that is, $(\bar{A}(r, s, t))^{-1}=\bar{A}\left(t, s^{\prime}, r\right)$, where $s^{\prime}=\left(s_{n}^{\prime}\right)$ such that $s_{n}^{\prime}=(-1)^{n} D_{n}^{(s)}$ for all $n \in \mathbb{N}[35]$, p.990. Therefore, we have by (2.1) that

$$
x_{n}=\frac{1}{t_{n}} \sum_{k=0}^{n}(-1)^{n-k} D_{n-k}^{(s)} r_{k} \bar{x}_{k} \quad(n \in \mathbb{N}) \text {. }
$$

For an arbitrary subset $X$ of $w$, the set $X(r, s, t)$ has recently been introduced in [35] as the matrix domain of the triangle $\bar{A}(r, s, t)$ in $X$, that is,

$$
X(r, s, t)=\left\{x=\left(x_{k}\right) \in w: y=\left(\frac{1}{r_{n}} \sum_{k=0}^{n} s_{n-k} t_{k} x_{k}\right)_{n=0}^{\infty} \in X\right\} .
$$

It is obvious that $X(r, s, t)$ is a sequence space whenever $X$ is a sequence space, and we call it the sequence space of generalized means. Further, if $X$ is a $B K$ space then $\bar{X}=X(r, s, t)$ is also a $B K$ space with the norm given by

$$
\|x\|_{\bar{X}}=\|y\|_{X} \quad(x \in \bar{X}) .
$$

Recently, Maji and Srivastava [36] have defined and studied the sequence space $X(r, s$, $\left.t ; B^{(m)}\right)$ for $X \in\left\{\ell_{\infty}, c, c_{0}\right\}$ which is obtained by combining the generalized means and the $m$ th order generalized difference operator $B^{(m)}(u, v)$. They characterized some compact operators on the spaces $X\left(r, s, t ; B^{(m)}\right)$ for $X \in\left\{\ell_{\infty}, c, c_{0}\right\}$ by using the Hausdorff measure of noncompactness. In this paper, we derive some identities or estimates for the operator norms and the Hausdorff measures of noncompactness of certain matrix operators on the sequence space $\ell_{p}\left(r, s, t ; B^{(m)}\right)$. By applying the Hausdorff measure of noncompactness, we obtain the necessary and sufficient conditions for such operators to be compact. Further, we study some geometric properties of this space.

The generalized difference matrix of order $m$ denoted as $B^{(m)}=B^{(m)}(u, v)=\left(b_{n k}^{(m)}\right), u, v \neq 0$ (see [12]) is defined as

$$
b_{n k}^{(m)}= \begin{cases}\left(\begin{array}{c}
m \\
n-k
\end{array}\right) u^{m-n+k} v^{n-k} & (\max \{0, n-m\} \leq k \leq n), \\
0 & (0 \leq k<\max \{0, n-m\}), \\
0 & (k>n) .\end{cases}
$$


The sequence spaces $X\left(r, s, t ; B^{(m)}\right)$ for $X \in\left\{\ell_{\infty}, c, c_{0}\right\}$ are defined as follows:

$$
X\left(r, s, t ; B^{(m)}\right)=\left\{x=\left(x_{k}\right) \in w:\left(\left(\bar{A}(r, s, t) \cdot B^{(m)}\right) x\right)_{n=0}^{\infty} \in X\right\} .
$$

By using the matrix domain, we can write

$$
X\left(r, s, t ; B^{(m)}\right)=X_{\bar{A}\left(r, s, t ; B^{(m)}\right)}=\left\{x=\left(x_{k}\right) \in w: \bar{A}\left(r, s, t ; B^{(m)}\right) x \in X\right\},
$$

where $\bar{A}\left(r, s, t ; B^{(m)}\right)=\bar{A}(r, s, t) \cdot B^{(m)}$. The sequence $\bar{x}=\left(\bar{x}_{n}\right)$ is $\bar{A}(r, s, t) \cdot B^{(m)}$-transform of $x=$ $\left(x_{n}\right)$, i.e.

$$
\bar{x}_{n}=\sum_{j=0}^{n}\left(\sum_{i=j}^{n}\left(\begin{array}{c}
m \\
i-j
\end{array}\right) \frac{s_{n-i} t_{i}}{r_{n}} u^{m+j-i} v^{i-j}\right) x_{j} \quad(n \in \mathbb{N}) .
$$

In this paper, we are interested in the study of $\ell_{p}\left(r, s, t ; B^{(m)}\right)$. We have the following lemma which is immediate by Theorem 4.5 in [35].

Lemma 2.1 If $a=\left(a_{k}\right) \in\left(\ell_{p}\left(r, s, t ; B^{(m)}\right)\right)^{\beta}$, then $\tilde{a}=\left(\tilde{a}_{k}\right) \in \ell_{p}^{\beta}\left(=\ell_{q}\right)$ and we have

$$
\sum_{k=0}^{\infty} a_{k} x_{k}=\sum_{k=0}^{\infty} \tilde{a}_{k} \bar{x}_{k}
$$

for all $x=\left(x_{k}\right) \in \bar{\ell}_{p}$ with $\bar{x}=\left(\bar{A}\left(r, s, t ; B^{(m)}\right)\right) x$, where

$$
\begin{aligned}
\tilde{a}_{k}= & r_{k}\left[\frac{a_{k}}{s_{0} t_{k} u^{m}}+\sum_{i=k}^{k+1}(-1)^{i-k} \frac{D_{i-k}^{(s)}}{t_{i}} \sum_{j=k+1}^{\infty}\left(\begin{array}{c}
m+j-i-1 \\
j-i
\end{array}\right) \frac{(-v)^{j-i}}{w^{j-i+m}} a_{j}\right. \\
& \left.+\sum_{i=k+2}^{\infty}(-1)^{i-k} \frac{D_{i-k}^{(s)}}{t_{i}} \sum_{j=i}^{\infty}\left(\begin{array}{c}
m+j-i-1 \\
j-i
\end{array}\right) \frac{(-v)^{j-i}}{w^{j-i+m}} a_{j}\right] .
\end{aligned}
$$

The following results will be needed in our study.

\section{Lemma 2.2 We have}

$$
\|a\|_{\bar{\ell}_{p}}^{*}=\|\tilde{a}\|_{\ell_{p}}^{*}
$$

for all $a=\left(a_{k}\right) \in \bar{\ell}_{p}^{\beta}$, where $\tilde{a}=\left(\tilde{a}_{k}\right)$ is the sequence defined by (2.7).

Proof Let $a=\left(a_{k}\right) \in \bar{\ell}_{p}^{\beta}$. Then it follows by Lemma 2.1 that $\tilde{a}=\left(\tilde{a}_{k}\right) \in \ell_{p}^{\beta}$ and the equality (2.6) holds for all sequences $x=\left(x_{k}\right) \in \bar{\ell}_{p}$ and $\bar{x}=\left(\bar{x}_{k}\right) \in \ell_{p}$ which are connected by the relation $\bar{x}=\left(\bar{A}\left(r, s, t ; B^{(m)}\right)\right) x$. Further, we see that $x \in S_{\bar{\ell}_{p}}$ if and only if $\bar{x} \in S_{\ell_{p}}$. Therefore, we derive from (1.2) and (2.6) that

$$
\|a\|_{\bar{\ell}_{p}}^{*}=\sup _{x \in S_{\bar{\ell}_{p}}}\left|\sum_{k=0}^{\infty} a_{k} x_{k}\right|=\sup _{\bar{x} \in S_{\ell_{p}}}\left|\sum_{k=0}^{\infty} \tilde{a}_{k} \bar{x}_{k}\right|=\|\tilde{a}\|_{\ell_{p}}^{*} .
$$

This concludes the proof. 
Remark 2.3 By combining Lemmas 1.1 and 2.2, we have the following:

(a) If $a \in\left(\ell_{1}\left(r, s, t ; B^{(m)}\right)\right)^{\beta}$, then $\|a\|_{\bar{\ell}_{1}}^{*}=\sup _{k}\left|\tilde{a}_{k}\right|<\infty$.

(b) If $a \in\left(\ell_{p}\left(r, s, t ; B^{(m)}\right)\right)^{\beta}$, then $\|a\|_{\bar{\ell}_{p}}^{*}=\left(\sum_{k=0}^{\infty}\left|\tilde{a}_{k}\right|^{q}\right)^{1 / q}<\infty$, where $q=p /(p-1)$ and $1<p<\infty$.

Throughout this paper, if $A=\left(a_{n k}\right)$ is an infinite matrix, we define the associated matrix $\tilde{A}=\left(\tilde{a}_{n k}\right)$ by

$$
\begin{aligned}
\tilde{a}_{n k}= & r_{k}\left[\frac{a_{n k}}{s_{0} t_{k} u^{m}}+\sum_{i=k}^{k+1}(-1)^{i-k} \frac{D_{i-k}^{(s)}}{t_{i}} \sum_{j=k+1}^{\infty}\left(\begin{array}{c}
m+j-i-1 \\
j-i
\end{array}\right) \frac{(-v)^{j-i}}{w^{j-i+m}} a_{n j}\right. \\
& \left.+\sum_{i=k+2}^{\infty}(-1)^{i-k} \frac{D_{i-k}^{(s)}}{t_{i}} \sum_{j=i}^{\infty}\left(\begin{array}{c}
m+j-i-1 \\
j-i
\end{array}\right) \frac{(-v)^{j-i}}{w^{j-i+m}} a_{n j}\right]
\end{aligned}
$$

provided the series on the right converge for all $n, k \in \mathbb{N}$, which is the case whenever $A_{n} \in$ $\left(\ell_{p}(r, s, t)\right)^{\beta}$ for all $n \in \mathbb{N}[35]$, Theorem 4.5. Then we have the following.

Lemma 2.4 Let $Y$ be a sequence space and $A=\left(a_{n k}\right)$ be an infinite matrix. If $A \in$ $\left(\ell_{p}\left(r, s, t ; B^{(m)}\right), Y\right)$, then $\tilde{A} \in\left(\ell_{p}, Y\right)$ such that $A x=\tilde{A} \bar{x}$ for all $x \in \ell_{p}(r, s, t)$ with $\bar{x}=$ $(\bar{A}(r, s, t)) x$, where $\tilde{A}=\left(\tilde{a}_{n k}\right)$ is the associated matrix defined by (2.8).

Proof Suppose that $A \in\left(\ell_{p}\left(r, s, t ; B^{(m)}\right), Y\right)$ and let $x \in \ell_{p}\left(r, s, t ; B^{(m)}\right)$. Then $A_{n} \in\left(\ell_{p}(r, s, t\right.$; $\left.\left.B^{(m)}\right)\right)^{\beta}$ for all $n \in \mathbb{N}$. Thus, it follows by Lemma 2.1 that $\tilde{A}_{n} \in \ell_{p}^{\beta}$ for all $n \in \mathbb{N}$ and the equality $A x=\tilde{A} \bar{x}$ holds which yields that $\tilde{A} \bar{x} \in Y$, where $\bar{x}$ is the sequence of generalized means of $x$, i.e., $\bar{x}=\left(\bar{A}\left(r, s, t ; B^{(m)}\right)\right) x$. Further, it is obvious by (2.4) and Remark 2.3 that every $\bar{x} \in \ell_{p}$ is the sequence of generalized means of some $x \in \ell_{p}\left(r, s, t ; B^{(m)}\right)$. Hence, we deduce that $\tilde{A} \in\left(\ell_{p}, Y\right)$. This completes the proof.

Finally, we conclude this section by the following results on operator norms.

Theorem 2.5 Let $A=\left(a_{n k}\right)$ an infinite matrix and $\tilde{A}=\left(\tilde{a}_{n k}\right)$ the associated matrix. If $A$ is in any of the classes $\left(\ell_{p}\left(r, s, t ; B^{(m)}\right), \ell_{\infty}\right),\left(\ell_{p}\left(r, s, t ; B^{(m)}\right), c\right)$ or $\left(\ell_{p}\left(r, s, t ; B^{(m)}\right), c_{0}\right)$, then

$$
\left\|L_{A}\right\|=\|A\|_{\left(\ell_{p}\left(r, s, t ; B^{(m)}\right), \ell_{\infty}\right)}=\sup _{n}\left\|\tilde{A}_{n}\right\|_{\ell_{p}}^{*}<\infty .
$$

Proof This is immediate by combining Lemmas 1.1 and 2.2 .

Theorem 2.6 If $A \in\left(\ell_{p}\left(r, s, t ; B^{(m)}\right), \ell_{1}\right)$, then

$$
\|A\|_{\left(\ell_{p}\left(r, s, t ; B^{(m)}\right), \ell_{1}\right)} \leq\left\|L_{A}\right\| \leq 4 \cdot\|A\|_{\left(\ell_{p}\left(r, s, t ; B^{(m)}\right), \ell_{1}\right)},
$$

where

$$
\|A\|_{\left(\ell_{p}\left(r, s, t ; B^{(m)}\right), \ell_{1}\right)}=\sup _{N \in \mathcal{F}}\left\|\sum_{n \in N} \tilde{A}_{n}\right\|_{\ell_{p}}^{*}<\infty .
$$

Proof This result follows from Lemmas 1.2 and 2.2. 
Theorem 2.7 If $A \in\left(\ell_{1}\left(r, s, t ; B^{(m)}\right), \ell_{p}\right)$, then

$$
\left\|L_{A}\right\|=\|A\|_{\left(\ell_{1}\left(r, s, t ; B^{(m)}\right), \ell_{p}\right)}=\sup _{k}\left(\sum_{n=0}^{\infty}\left|\tilde{a}_{n k}\right|^{p}\right)^{1 / p}<\infty .
$$

Proof The proof is elementary and left to the reader.

Remark 2.8 The characterizations of matrix classes considered in this paper can easily be obtained as in Corollaries 5.4 and 5.7 of [35]. Thus, we shall omit these characterizations and only deal with the operator norms and the Hausdorff measures of noncompactness of some matrix operators which are given by infinite matrices in such classes.

\section{Main results}

In this section, we derive some identities or estimates for the Hausdorff measures of noncompactness of certain matrix operators on the spaces of generalized means. Further, we apply our results to obtain the necessary and sufficient (or only sufficient) conditions for such operators to be compact.

We may begin with quoting the following lemma [28], Theorem 3.7.

Lemma 3.1 Let $X \supset \phi$ be a BK space. Then we have:

(a) If $A \in\left(X, \ell_{\infty}\right)$, then

$$
0 \leq\left\|L_{A}\right\|_{\chi} \leq \limsup _{n \rightarrow \infty}\left\|A_{n}\right\|_{X}^{*}
$$

(b) If $A \in\left(X, c_{0}\right)$, then

$$
\left\|L_{A}\right\|_{\chi}=\limsup _{n \rightarrow \infty}\left\|A_{n}\right\|_{X}^{*}
$$

(c) If $X$ has $A K$ or $X=\ell_{\infty}$ and $A \in(X, c)$, then

$$
\frac{1}{2} \cdot \limsup _{n \rightarrow \infty}\left\|A_{n}-\alpha\right\|_{X}^{*} \leq\left\|L_{A}\right\|_{\chi} \leq \limsup _{n \rightarrow \infty}\left\|A_{n}-\alpha\right\|_{X}^{*},
$$

where $\alpha=\left(\alpha_{k}\right)$ with $\alpha_{k}=\lim _{n \rightarrow \infty} a_{n k}$ for all $k \in \mathbb{N}$.

Now, let $A=\left(a_{n k}\right)$ be an infinite matrix and $\tilde{A}=\left(\tilde{a}_{n k}\right)$ the associated matrix defined by (2.8). Then, by combining Lemmas $2.2,2.4$ and 3.1, we have the following result.

Theorem 3.2 We have:

(a) If $A \in\left(\ell_{p}\left(r, s, t ; B^{(m)}\right), \ell_{\infty}\right)$, then

$$
0 \leq\left\|L_{A}\right\|_{\chi} \leq \limsup _{n \rightarrow \infty}\left\|\tilde{A}_{n}\right\|_{\ell_{p}}^{*}
$$

and

$$
L_{A} \text { is compact if } \lim _{n \rightarrow \infty}\left\|\tilde{A}_{n}\right\|_{\ell_{p}}^{*}=0 \text {. }
$$


(b) If $A \in\left(\ell_{p}\left(r, s, t ; B^{(m)}\right), c_{0}\right)$, then

$$
\left\|L_{A}\right\|_{\chi}=\limsup _{n \rightarrow \infty}\left\|\tilde{A}_{n}\right\|_{\ell_{p}}^{*}
$$

and

$$
L_{A} \text { is compact if and only if } \lim _{n \rightarrow \infty}\left\|\tilde{A}_{n}\right\|_{\ell_{p}}^{*}=0 \text {. }
$$

(c) If $A \in\left(\ell_{p}\left(r, s, t ; B^{(m)}\right), c\right)$, then

$$
\frac{1}{2} \cdot \limsup _{n \rightarrow \infty}\left\|\tilde{A}_{n}-\tilde{\alpha}\right\|_{\ell_{p}}^{*} \leq\left\|L_{A}\right\|_{\chi} \leq \limsup _{n \rightarrow \infty}\left\|\tilde{A}_{n}-\tilde{\alpha}\right\|_{\ell_{p}}^{*}
$$

and

$$
L_{A} \text { is compact if and only if } \lim _{n \rightarrow \infty}\left\|\tilde{A}_{n}-\tilde{\alpha}\right\|_{\ell_{p}}^{*}=0 \text {, }
$$

where $\tilde{\alpha}=\left(\tilde{\alpha}_{k}\right)$ with $\tilde{\alpha}_{k}=\lim _{n \rightarrow \infty} \tilde{a}_{n k}$ for all $k \in \mathbb{N}$.

Proof Note that parts (a) and (b) are proved in [37]. Further it is obvious that (3.2), (3.4) and (3.6) are, respectively, obtained from (3.1), (3.3), and (3.5) by using (1.2). Thus, we have to prove (3.1), (3.3) and (3.5).

Since $\ell_{p}\left(r, s, t ; B^{(m)}\right)$ is a $B K$ space, we deduce by means of Lemma 2.2 that (3.1) and (3.3) are immediate by parts (a) and (b) of Lemma 3.1, respectively.

To prove (3.5), we have $A \in\left(\ell_{p}\left(r, s, t ; B^{(m)}\right), c\right)$ and hence $\tilde{A} \in(X, c)$ by Lemma 2.4. Therefore, it follows by part (c) of Lemma 3.1 that

$$
\frac{1}{2} \cdot \limsup _{n \rightarrow \infty}\left\|\tilde{A}_{n}-\tilde{\alpha}\right\|_{X}^{*} \leq\left\|L_{\tilde{A}}\right\|_{\chi} \leq \limsup _{n \rightarrow \infty}\left\|\tilde{A}_{n}-\tilde{\alpha}\right\|_{X}^{*}
$$

where $\tilde{\alpha}=\left(\tilde{\alpha}_{k}\right)$ and $\tilde{\alpha}_{k}=\lim _{n \rightarrow \infty} \tilde{a}_{n k}$ for all $k \in \mathbb{N}$.

Now, let us write $S=S_{X}$ and $\bar{S}=S_{\ell_{p}\left(r, s, t ; B^{(m)}\right)}$, for short. Then we obtain by (1.3) and Lemma 1.1

$$
\left\|L_{A}\right\|_{\chi}=\chi\left(L_{A}(\bar{S})\right)=\chi(A \bar{S})
$$

and

$$
\left\|L_{\tilde{A}}\right\|_{\chi}=\chi\left(L_{\tilde{A}}(S)\right)=\chi(\tilde{A} S)
$$

Further, we see that $x \in \bar{S}$ if and only if $\bar{x} \in S$, and since $A x=\tilde{A} \bar{x}$ by Lemma 2.4 , we deduce that $A \bar{S}=\tilde{A} S$. This leads us with (3.8) and (3.9) to the consequence that $\left\|L_{A}\right\|_{\chi}=\left\|L_{\tilde{A}}\right\|_{\chi}$. Hence, we get (3.5) from (3.7). This completes the proof.

It is worth mentioning that the condition in (3.2) is only a sufficient condition for the operator $L_{A}$ to be compact, where $A \in\left(\ell_{p}\left(r, s, t ; B^{(m)}\right), \ell_{\infty}\right)$ and $X$ is a $B K$ space with $A K$ or $X=\ell_{\infty}$. More precisely, the following example will show that it is possible for $L_{A}$ to be compact while $\lim _{n \rightarrow \infty}\left\|\tilde{A}_{n}\right\|_{X}^{*} \neq 0$. Hence, in general, we have just 'if' in (3.2) of Theorem 3.2(a). 
Example 3.3 Let us define the matrix $A=\left(a_{n k}\right)$ by $a_{n 0}=s_{0} t_{0} / r_{0}$ and $a_{n k}=0$ for $k \geq 1$ $(n \in \mathbb{N})$. Let $B^{(m)}=I$, the identity matrix. Then we have for every $x=\left(x_{k}\right) \in \ell_{p}\left(r, s, t ; B^{(m)}\right)$, $A x=\left(s_{0} t_{0} x_{0} / r_{0}\right) e$ and hence $A \in\left(\ell_{p}\left(r, s, t ; B^{(m)}\right), \ell_{\infty}\right)$. Further, it is obvious that $L_{A}$ is of finite rank and so $L_{A}$ is compact. On the other hand, by using (2.8), it can easily be seen that $\tilde{A}_{n}=e^{(0)}$ for all $n \in \mathbb{N}$. Thus, we obtain by Lemma 1.1 that $\left\|\tilde{A}_{n}\right\|_{X}^{*}=1$ for all $n \in \mathbb{N}$, which implies that $\lim _{n \rightarrow \infty}\left\|\tilde{A}_{n}\right\|_{X}^{*}=1$.

Moreover, as an immediate consequence of Theorem 3.2, we have the following corollary.

Corollary 3.4 If either $A \in\left(\ell_{\infty}\left(r, s, t ; B^{(m)}\right), c_{0}\right)$ or $A \in\left(\ell_{\infty}\left(r, s, t ; B^{(m)}\right), c\right)$, then the operator $L_{A}$ is compact.

Proof Let $A \in\left(\bar{\ell}_{\infty}, c_{0}\right)$. Then we have by Lemma 2.4 that $\tilde{A} \in\left(\ell_{\infty}, c_{0}\right)$ which implies that $\lim _{n \rightarrow \infty}\left(\sum_{k=0}^{\infty}\left|\tilde{a}_{n k}\right|\right)=0$, that is, $\lim _{n \rightarrow \infty}\left\|\tilde{A}_{n}\right\|_{\ell_{\infty}}^{*}=0$ by Lemma 1.1. This leads us with Theorem 3.2(b) to the consequence that $L_{A}$ is compact. Similarly, if $A \in\left(\ell_{\infty}\left(r, s, t ; B^{(m)}\right), c\right)$ then $\tilde{A} \in\left(\ell_{\infty}, c\right)$ and hence $\lim _{n \rightarrow \infty}\left(\sum_{k=0}^{\infty}\left|\tilde{a}_{n k}-\tilde{\alpha}_{k}\right|\right)=0$, which can be written as $\lim _{n \rightarrow \infty} \| \tilde{A}_{n}-$ $\tilde{\alpha} \|_{\ell_{\infty}}^{*}=0$, where $\tilde{\alpha}=\left(\tilde{\alpha}_{k}\right)$ and $\tilde{\alpha}_{k}=\lim _{n \rightarrow \infty} \tilde{a}_{n k}$ for all $k \in \mathbb{N}$. Therefore, we deduce from Theorem 3.2(c) that $L_{A}$ is compact.

Throughout, let $\mathcal{F}_{r}(r \in \mathbb{N})$ be the subcollection of $\mathcal{F}$ consisting of all non-empty and finite subsets of $\mathbb{N}$ with elements that are greater than $r$, that is,

$$
\mathcal{F}_{r}=\{N \in \mathcal{F}: n>r \text { for all } n \in N\} \quad(r \in \mathbb{N})
$$

Then we have the following.

Lemma 3.5 (Theorem 3.11 in [28]) Let $X \supset \phi$ be a $B K$ space. If $A \in\left(X, \ell_{1}\right)$, then

$$
\lim _{r \rightarrow \infty}\left(\sup _{N \in \mathcal{F}_{r}}\left\|\sum_{n \in N} A_{n}\right\|_{X}^{*}\right) \leq\left\|L_{A}\right\|_{\chi} \leq 4 \cdot \lim _{r \rightarrow \infty}\left(\sup _{N \in \mathcal{F}_{r}}\left\|\sum_{n \in N} A_{n}\right\|_{X}^{*}\right)
$$

Theorem 3.6 If $A \in\left(\ell_{p}\left(r, s, t ; B^{(m)}\right), \ell_{1}\right)$, then

$$
\lim _{r \rightarrow \infty}\|A\|_{\left(\ell_{p}\left(r, s, t ; B^{(m)}\right), \ell_{1}\right)}^{(r)} \leq\left\|L_{A}\right\|_{\chi} \leq 4 \cdot \lim _{r \rightarrow \infty}\|A\|_{\left(\ell_{p}\left(r, s, t ; B^{(m)}\right), \ell_{1}\right)}^{(r)}
$$

and

$$
L_{A} \text { is compact if and only if } \lim _{r \rightarrow \infty}\|A\|_{\left(\ell_{p}\left(r, s, t ; B^{(m)}\right), \ell_{1}\right)}^{(r)}=0,
$$

where

$$
\|A\|_{\left(\ell_{p}\left(r, s, t ; B^{(m)}\right), \ell_{1}\right)}^{(r)}=\sup _{N \in \mathcal{F}_{r}}\left\|\sum_{n \in N} \tilde{A}_{n}\right\|_{X}^{*} \quad(r \in \mathbb{N})
$$

Proof It is obvious that (3.10) is obtained by combining Lemmas 2.2 and 3.5. Also, by using (1.2), we get (3.11) from (3.10). 
Corollary 3.7 Let $1<p<\infty, q=p /(p-1)$. If $A \in\left(\ell_{p}\left(r, s, t ; B^{(m)}\right), \ell_{1}\right)$, then

$$
\lim _{r \rightarrow \infty}\|A\|_{\left(\ell_{p}\left(r, s, t ; B^{(m)}\right), \ell_{1}\right)}^{(r)} \leq\left\|L_{A}\right\|_{\chi} \leq 4 \cdot \lim _{r \rightarrow \infty}\|A\|_{\left(\ell_{p}\left(r, s, t ; B^{(m)}\right), \ell_{1}\right)}^{(r)}
$$

and

$$
L_{A} \text { is compact if and only if } \lim _{r \rightarrow \infty}\|A\|_{\left(\ell_{p}\left(r, s, t ; B^{(m)}\right), \ell_{1}\right)}^{(r)}=0 \text {, }
$$

where

$$
\|A\|_{\left(\ell_{p}\left(r, s, t ; B^{(m)}\right), \ell_{1}\right)}^{(r)}=\sup _{N \in \mathcal{F}_{r}}\left(\sum_{k=0}^{\infty}\left|\sum_{n \in N} \tilde{a}_{n k}\right|^{q}\right)^{1 / q} \quad(r \in \mathbb{N}) .
$$

Now, we prove the following result.

Theorem 3.8 Let $1 \leq p<\infty$. If $A \in\left(\ell_{1}\left(r, s, t ; B^{(m)}\right), \ell_{p}\right)$, then

$$
\left\|L_{A}\right\|_{\chi}=\lim _{r \rightarrow \infty}\left(\sup _{k}\left(\sum_{n=r}^{\infty}\left|\tilde{a}_{n k}\right|^{p}\right)^{1 / p}\right)
$$

and

$$
L_{A} \text { is compact if and only if } \lim _{r \rightarrow \infty}\left(\sup _{k}\left(\sum_{n=r}^{\infty}\left|\tilde{a}_{n k}\right|^{p}\right)\right)=0 \text {. }
$$

Proof We write $\bar{S}=S_{\bar{\ell}_{1}}$. Then we see by Lemma 1.1 that $L_{A}(\bar{S})=A \bar{S} \in \mathcal{M}_{\ell_{p}}$. Thus, it follows from (1.3) and Lemma 1.3 that

$$
\left\|L_{A}\right\|_{\chi}=\chi(A \bar{S})=\lim _{r \rightarrow \infty}\left(\sup _{x \in \bar{S}}\left\|\left(I-P_{r}\right)(A x)\right\|_{\ell_{p}}\right)
$$

where $P_{r}: \ell_{p} \rightarrow \ell_{p}(r \in \mathbb{N})$ is the operator defined by $P_{r}(x)=\left(x_{0}, x_{1}, \ldots, x_{r}, 0,0, \ldots\right)$ for all $x=\left(x_{k}\right) \in \ell_{p}$ and $I$ is the identity operator on $\ell_{p}$.

On the other hand, let $x \in \ell_{1}\left(r, s, t ; B^{(m)}\right)$ be given. Then $\bar{x} \in \ell_{1}$ and since $A \in\left(\ell_{1}(r, s, t\right.$; $\left.\left.B^{(m)}\right), \ell_{p}\right)$, we obtain from Lemma 2.4 that $\tilde{A} \in\left(\ell_{1}, \ell_{p}\right)$ and $A x=\tilde{A} \bar{x}$. Thus, we have for every $r \in \mathbb{N}$,

$$
\begin{aligned}
\left\|\left(I-P_{r}\right)(A x)\right\|_{\ell_{p}} & =\left\|\left(I-P_{r}\right)(\tilde{A} \bar{x})\right\|_{\ell_{p}} \\
& =\left(\sum_{n=r+1}^{\infty}\left|\tilde{A}_{n}(\bar{x})\right|^{p}\right)^{1 / p} \\
& =\left(\sum_{n=r+1}^{\infty}\left|\sum_{k=0}^{\infty} \tilde{a}_{n k} \bar{x}_{k}\right|^{p}\right)^{1 / p} \\
& \leq \sum_{k=0}^{\infty}\left(\sum_{n=r+1}^{\infty}\left|\tilde{a}_{n k} \bar{x}_{k}\right|^{p}\right)^{1 / p}
\end{aligned}
$$




$$
\begin{aligned}
& \leq\|\bar{x}\|_{\ell_{1}}\left(\sup _{k}\left(\sum_{n=r+1}^{\infty}\left|\tilde{a}_{n k}\right|^{p}\right)^{1 / p}\right) \\
& =\|x\|_{\ell_{1}(r, s, t)}\left(\sup _{k}\left(\sum_{n=r+1}^{\infty}\left|\tilde{a}_{n k}\right|^{p}\right)^{1 / p}\right) .
\end{aligned}
$$

This yields

$$
\sup _{x \in \bar{S}}\left\|\left(I-P_{r}\right)(A x)\right\|_{\ell_{p}} \leq \sup _{k}\left(\sum_{n=r+1}^{\infty}\left|\tilde{a}_{n k}\right|^{p}\right)^{1 / p} \quad(r \in \mathbb{N}) .
$$

Therefore, we deduce from (3.14) that

$$
\left\|L_{A}\right\|_{\chi} \leq \lim _{r \rightarrow \infty}\left(\sup _{k}\left(\sum_{n=r+1}^{\infty}\left|\tilde{a}_{n k}\right|^{p}\right)^{1 / p}\right) .
$$

To prove the converse inequality, let $b^{(k)} \in \ell_{1}\left(r, s, t ; B^{(m)}\right)$ be such that $\left(\bar{A}\left(r, s, t ; B^{(m)}\right)\right) b^{(k)}=$ $e^{(k)}(k \in \mathbb{N})$, that is, $e^{(k)}$ is the sequence of generalized means of $b^{(k)}$ for each $k \in \mathbb{N}$ (see Corollary 3.5 in [35]). Then we have by Lemma 2.4 that $A b^{(k)}=\tilde{A} e^{(k)}=\left(\tilde{a}_{n k}\right)_{n=0}^{\infty}$ for every $k \in \mathbb{N}$.

Now, let $B=\left\{b^{(k)}: k \in \mathbb{N}\right\}$. Then $B \subset \bar{S}$ and hence $A B \subset A \bar{S}$, which implies that $\chi(A B) \leq$ $\chi(A \bar{S})=\left\|L_{A}\right\|_{\chi}$.

Further, it follows by applying Lemma 1.3 that

$$
\begin{aligned}
\chi(A B) & =\lim _{r \rightarrow \infty}\left(\sup _{k}\left(\sum_{n=r+1}^{\infty}\left|A_{n}\left(b^{(k)}\right)\right|^{p}\right)^{1 / p}\right) \\
& =\lim _{r \rightarrow \infty}\left(\sup _{k}\left(\sum_{n=r+1}^{\infty}\left|\tilde{a}_{n k}\right|^{p}\right)^{1 / p}\right) .
\end{aligned}
$$

Thus, we obtain

$$
\lim _{r \rightarrow \infty}\left(\sup _{k}\left(\sum_{n=r+1}^{\infty}\left|\tilde{a}_{n k}\right|^{p}\right)^{1 / p}\right) \leq\left\|L_{A}\right\|_{\chi} .
$$

Hence, we get (3.12) by combining (3.15) and (3.16). This completes the proof, since (3.13) is immediate by (1.2) and (3.12).

Finally, we end this section with the following example, which shows that the limit in (3.12) may not be zero, that is, there exist matrix operators in the class $\mathcal{B}\left(\bar{\ell}_{1}, \ell_{p}\right)$ which are not compact, where $1 \leq p<\infty$.

Example 3.9 Let $A=\left(a_{n k}\right)$ be the infinite matrix defined by $A=\bar{A}\left(r, s, t ; B^{(m)}\right)$. Let $B^{(m)}=$ $I$, the identity matrix. Since $\ell_{1}\left(r, s, t ; B^{(m)}\right)$ is the matrix domain of $A$ in $\ell_{1}$, we have $A \in$ $\left(\ell_{1}\left(r, s, t ; B^{(m)}\right), \ell_{1}\right)$ and hence $A \in\left(\bar{\ell}_{1}, \ell_{p}\right)$ for $1 \leq p<\infty$. Further, it is trivial to see that the associated matrix $\tilde{A}$ is the identity matrix, that is, $\tilde{a}_{n n}=1$ and $\tilde{a}_{n k}=0$ for $k \neq n(n \in \mathbb{N})$. 
Now, let $r \in \mathbb{N}$ be given. Then we have, for every $k \in \mathbb{N}$,

$$
\sum_{n=r}^{\infty}\left|\tilde{a}_{n k}\right|^{p}= \begin{cases}1 & (k \geq r), \\ 0 & (k<r) .\end{cases}
$$

This implies that

$$
\sup _{k}\left(\sum_{n=r}^{\infty}\left|\tilde{a}_{n k}\right|^{p}\right)^{1 / p}=1 \quad(r \in \mathbb{N})
$$

which leads us with (3.12) of Theorem 3.8 to the conclusion that $\left\|L_{A}\right\|_{\chi}=1$ and hence $L_{A}$ is not compact.

\section{Geometric properties}

Recently there has been a lot of interest in investigating geometric properties of sequence spaces besides topological and some other usual properties. In the literature, there are many papers concerning geometric properties of various Banach sequence spaces.

A Banach space $X$ is said to be a Köthe sequence space (see [15, 17], and [21]) if $X$ is a subspace of $w$ such that

(i) if $x \in w, y \in X$, and $|x(i)| \leq|y(i)|$ for all $i \in \mathbb{N}$, then $x \in X$ and $\|x\| \leq\|y\|$;

(ii) there exists an element $x \in X$ such that $x(i)>0$ for all $i \in \mathbb{N}$.

A Köthe sequence space $X$ is said to have the Fatou property if for any real sequence $x$ and any $\left\{x_{n}\right\}$ in $X$ such that $x_{n} \rightarrow x$ coordinatewise and $\sup _{n}\left\|x_{n}\right\|<\infty$, we have $x \in X$ and $\left\|x_{n}\right\| \rightarrow\|x\|$.

A Banach space $X$ is said to have the Banach-Saks property if every bounded sequence $\left\{x_{n}\right\}$ in $X$ admits a subsequence $\left\{z_{n}\right\}$ such that the sequence $\left\{t_{k}(z)\right\}$ is convergent in $X$ with respect to the norm, where

$$
t_{k}(z)=\frac{1}{k}\left(z_{1}+z_{2}+\cdots+z_{k}\right) \quad \forall k \in \mathbb{N} .
$$

A Banach space $X$ is said to have the weak Banach-Saks property whenever given any weakly null sequence $\left\{x_{n}\right\}$ in $X$ there exists its subsequence $\left\{z_{n}\right\}$ such that the sequence $\left\{t_{k}(z)\right\}$ converges to zero strongly.

Given any $p \in(1, \infty)$, we say that a Banach space $(X,\|\cdot\|)$ has the Banach-Saks property of type $p$ if there exists a constant $c>0$ such that every weakly null sequence $\left\{x_{k}\right\}$ has a subsequence $\left\{x_{k_{\ell}}\right\}$ such that (see [22])

$$
\left\|\sum_{\ell=1}^{n} x_{k \ell}\right\| \leq c n^{\frac{1}{p}} \quad \forall n \in \mathbb{N} .
$$

The Banach-Saks property of type $p \in(1, \infty)$ and weak Banach-Saks property for Cesàro sequence spaces have been considered in [15].

We say that a Banach space $X$ has the weak fixed point property if every nonexpansive self-mapping defined on a non-empty weakly compact convex subset of $X$ has a fixed point. 
In [18], García-Falset introduced the following coefficient for a Banach space $(X,\|\cdot\|)$ :

$$
R(X)=\sup \left\{\liminf _{n \rightarrow \infty}\left\|x_{n}-x\right\|: x \in B(X),\left\{x_{n}\right\} \subset B(X) \text { and } x_{n} \rightarrow 0 \text { weakly }\right\}
$$

and he proved (see $[18,19])$ that a Banach space $X$ with $R(X)<2$ has the weak fixed point property.

A Köthe sequence space $X$ is said to be order continuous if for any sequence $\left\{x_{n}\right\}$ and any $x$ in $X_{+}$(the positive cone in $X$ ) such that $x_{n}(i) \leq x(i)$ as $n \rightarrow \infty$ for all $i, n \in \mathbb{N}$ and $x_{n}(i) \rightarrow 0$ for any $i \in \mathbb{N}$, we have $\left\|x_{n}\right\| \rightarrow 0$ as $n \rightarrow \infty$.

Clarkson modulus of convexity of a normed space $(X,\|\cdot\|)$ is defined (see work by Clarkson [14] and Day [16]) by the formula

$$
\delta_{X}(\varepsilon)=\inf \left\{1-\frac{\|x+y\|}{2} ; x, y \in S(X),\|x-y\|=\varepsilon\right\}
$$

for any $\varepsilon \in[0,2]$. The inequality $\delta_{X}(\varepsilon)>0$ for all $\varepsilon \in(0,2]$ characterizes the uniform convexity of $X$ and the equality $\delta_{X}(2)=1$ characterizes strict convexity (= rotundity) of $X$.

The Gurarii modulus of convexity of a normed space $X$ is defined (see [20]) by

$$
\beta_{X}(\varepsilon)=\inf \left\{1-\inf _{\alpha \in[0,1]}\|\alpha x+(1-\alpha) y\| ; x, y \in \delta(X),\|x-y\|=\varepsilon\right\}
$$

for any $\varepsilon \in[0,2]$. It is obvious that $\delta_{X}(\varepsilon) \leq \beta_{X}(\varepsilon)$ for any Banach space $X$ and any $\varepsilon \in[0,2]$. It is also known that $\beta_{X}(\varepsilon) \leq 2 \delta_{X}(\varepsilon)$ for any $\varepsilon \in[0,2]$ and that $X$ is rotund if and only if $\beta_{X}(\varepsilon)=2$ and $X$ is uniformly convex if and only if $\beta_{X}(\varepsilon)>0$ for any $\varepsilon \in[0,2]$.

Theorem 4.1 The Banach-Saks type of the space $\ell_{p}\left(r, s, t ; B^{(m)}\right)$ is equal to $p$.

Proof Let $\left(\varepsilon_{n}\right)$ be a sequence of positive numbers for which $\sum_{n=1}^{\infty} \varepsilon_{n} \leq \frac{1}{2}$. Let $\left\{x_{n}\right\}$ be a weakly null sequence in $\ell_{p}\left(r, s, t ; B^{(m)}\right)$. Set $t_{0}=x_{0}=0$ and $t_{1}=x_{n_{1}}=x_{1}$. Then there exists $r_{1} \in \mathbb{N}$ such that

$$
\left\|\sum_{i=r_{1}+1}^{\infty} t_{1}(i) e^{(i)}\right\|_{\ell_{p}\left(r, s, t ; B^{(m)}\right)}<\varepsilon_{1} .
$$

Since the fact that $\left\{x_{n}\right\}$ is a weakly null sequence implies that $x_{n} \rightarrow 0$, coordinatewise, there is an $n_{2} \in \mathbb{N}$ such that

$$
\left\|\sum_{i=0}^{r_{1}} x_{n}(i) e^{(i)}\right\|_{\ell_{p}\left(r, s, t ; B^{(m)}\right)}<\varepsilon_{1}
$$

for all $n \geq n_{2}$. Set $t_{2}=x_{n_{2}}$. Then there exists an $r_{2}>r_{1}$ such that

$$
\left\|\sum_{i=r_{2}+1}^{\infty} t_{2}(i) e^{(i)}\right\|_{\ell_{p}\left(r, s, t ; B^{(m)}\right)}<\varepsilon_{2} .
$$


By using the fact that $x_{n} \rightarrow 0$ coordinatewise, there exists an $n_{3}>n_{2}$ such that

$$
\left\|\sum_{i=0}^{r_{2}} x_{n}(i) e^{(i)}\right\|_{\ell_{p}\left(r, s, t ; B^{(m)}\right)}<\varepsilon_{2}
$$

for all $n \geq n_{3}$. Continuing this process, we can find by induction two increasing subsequences $\left(r_{i}\right)$ and $\left(n_{i}\right)$ such that

$$
\left\|\sum_{i=0}^{r_{j}} x_{n}(i) e^{(i)}\right\|_{\ell_{p}\left(r, s, t ; B^{(m)}\right)}<\varepsilon_{j}
$$

for all $n \geq n_{j+1}$ and

$$
\left\|\sum_{i=r_{j}+1}^{\infty} t_{j}(i) e^{(i)}\right\|_{\ell_{p}\left(r, s, t ; B^{(m)}\right)}<\varepsilon_{j},
$$

where $t_{j}=x_{n_{j}}$. Hence,

$$
\begin{aligned}
\left\|\sum_{j=0}^{n} t_{j}\right\|_{\ell_{p}\left(r, s, t ; B^{(m)}\right)} & \left\|\sum_{j=0}^{n}\left(\sum_{i=0}^{r_{j-1}} t_{j}(i) e^{(i)}+\sum_{i=r_{j-1}+1}^{r_{j}} t_{j}(i) e^{(i)}+\sum_{i=r_{j}+1}^{\infty} t_{j}(i) e^{(i)}\right)\right\|_{\ell_{p}\left(r, s, t ; B^{(m)}\right)} \\
\leq & \left\|\sum_{j=0}^{n}\left(\sum_{i=r_{j-1}+1}^{r_{j}} t_{j}(i) e^{(i)}\right)\right\|_{\ell_{p}\left(r, s, t ; B^{(m)}\right)}+\left\|\sum_{j=0}^{n}\left(\sum_{i=0}^{r_{j-1}} t_{j}(i) e^{(i)}\right)\right\|_{\ell_{p}(u, v)} \\
& +\left\|\sum_{j=0}^{n}\left(\sum_{i=r_{j}+1}^{\infty} t_{j}(i) e^{(i)}\right)\right\|_{\ell_{p}\left(r, s, t ; B^{(m)}\right)} \\
\leq & \left\|\sum_{j=0}^{n}\left(\sum_{i=r_{j-1}+1}^{r_{j}} t_{j}(i) e^{(i)}\right)\right\|_{\ell_{p}\left(r, s, t ; B^{(m)}\right)}+2 \sum_{j=0}^{n} \varepsilon_{j}
\end{aligned}
$$

and

$$
\begin{aligned}
\left\|\sum_{j=0}^{n}\left(\sum_{i=r_{j-1}+1}^{r_{j}} t_{j}(i) e^{(i)}\right)\right\|_{\ell_{p}\left(r, s, t ; B^{(m)}\right)}^{p} & =\sum_{j=0}^{n} \sum_{i=r_{j-1}+1}^{r_{j}}\left|\sum_{k=0}^{i} u_{i} v_{k} t_{j}(k)\right|^{p} \\
& \leq \sum_{j=0}^{n} \sum_{i=0}^{\infty}\left|\sum_{k=0}^{i} u_{i} v_{k} t_{j}(k)\right|^{p} \\
& \leq(n+1) .
\end{aligned}
$$

Hence we obtain

$$
\left\|\sum_{j=0}^{n}\left(\sum_{i=r_{j-1}+1}^{r_{j}} t_{j}(i) e^{(i)}\right)\right\| \leq\left(\sum_{j=0}^{n} 1\right)^{\frac{1}{p}}=(n+1)^{\frac{1}{p}} .
$$


By using the inequality $1 \leq(n+1)^{\frac{1}{p}}$ for all $n \in \mathbb{N}$ and $1 \leq p<\infty$, we have

$$
\left\|\sum_{j=0}^{n} t_{j}\right\|_{\ell_{p}\left(r, s, t ; B^{(m)}\right)} \leq(n+1)^{\frac{1}{p}}+1 \leq 2(n+1)^{\frac{1}{p}}
$$

Therefore, the space $\ell_{p}\left(r, s, t ; B^{(m)}\right)$ has the Banach-Saks type $p$, which completes the proof of the theorem.

Theorem 4.2 The Gurarii of modulus of convexity for the normed space $\ell_{p}\left(r, s, t ; B^{(m)}\right)$ satisfies the inequality

$$
\beta_{\ell_{p}\left(r, s, t ; B^{(m)}\right)}(\varepsilon) \leq 1-\left(1-\left(\frac{\varepsilon}{2}\right)^{p}\right)^{\frac{1}{p}}
$$

for any $0 \leq \varepsilon \leq 2$.

Proof Let $x \in \ell_{p}\left(r, s, t ; B^{(m)}\right)$. By using (1.1), we have

$$
\|x\|_{\ell_{p}\left(r, s, t ; B^{(m)}\right)}=\left\|\left(\bar{A}(r, s, t) \cdot B^{(m)}\right) x\right\|_{\ell_{p}}=\left(\sum_{n}\left|\left(\left(\bar{A}(r, s, t) \cdot B^{(m)}\right) x\right)_{n}\right|^{p}\right)^{\frac{1}{p}} .
$$

Let $0 \leq \varepsilon \leq 2$ and using (1.2), let us consider the following sequences:

$$
\begin{aligned}
& x=\left(x_{n}\right)=\left(H\left(\left(1-\left(\frac{\varepsilon}{2}\right)^{p}\right)^{\frac{1}{p}}\right), H\left(\frac{\varepsilon}{2}\right), 0,0, \ldots\right), \\
& t=\left(t_{n}\right)=\left(H\left(\left(1-\left(\frac{\varepsilon}{2}\right)^{p}\right)^{\frac{1}{p}}\right), H\left(-\frac{\varepsilon}{2}\right), 0,0, \ldots\right),
\end{aligned}
$$

where $H=\left(\bar{A}(r, s, t) \cdot B^{(m)}\right)_{n k}^{-1}$. Since $y_{n}=\left(\left(\bar{A}(r, s, t) \cdot B^{(m)}\right) x\right)_{n}$ and $z_{n}=\left(\left(\bar{A}(r, s, t) \cdot B^{(m)}\right) t\right)_{n}$, we have

$$
\begin{aligned}
& y=\left(y_{n}\right)=\left(\left(1-\left(\frac{\varepsilon}{2}\right)^{p}\right)^{\frac{1}{p}},\left(\frac{\varepsilon}{2}\right), 0,0, \ldots\right), \\
& z=\left(z_{n}\right)=\left(\left(1-\left(\frac{\varepsilon}{2}\right)^{p}\right)^{\frac{1}{p}},\left(-\frac{\varepsilon}{2}\right), 0,0, \ldots\right) .
\end{aligned}
$$

By using the sequences given above, we obtain the following equalities:

$$
\begin{aligned}
\|x\|_{\ell_{p}\left(r, s, t ; B^{(m)}\right)}^{p} & =\left\|\left(\bar{A}(r, s, t) \cdot B^{(m)}\right) x\right\|_{\ell_{p}}^{p}=\left|\left(1-\left(\frac{\varepsilon}{2}\right)^{p}\right)^{\frac{1}{p}}\right|^{p}+\left|\frac{\varepsilon}{2}\right|^{p} \\
& =1-\left(\frac{\varepsilon}{2}\right)^{p}+\left(\frac{\varepsilon}{2}\right)^{p} \\
& =1, \\
\|t\|_{\ell_{p}\left(r, s, t ; B^{(m)}\right)}^{p} & =\left\|\left(\bar{A}(r, s, t) \cdot B^{(m)}\right) t\right\|_{\ell_{p}}^{p}=\left|\left(1-\left(\frac{\varepsilon}{2}\right)^{p}\right)^{\frac{1}{p}}\right|^{p}+\left|-\frac{\varepsilon}{2}\right|^{p}
\end{aligned}
$$




$$
\begin{aligned}
=1 & -\left(\frac{\varepsilon}{2}\right)^{p}+\left(\frac{\varepsilon}{2}\right)^{p} \\
=1 & \\
\|x-t\|_{\ell_{p}\left(r, s, t ; B^{(m)}\right)} & =\left\|\left(\bar{A}(r, s, t) \cdot B^{(m)}\right) x-\left(\bar{A}(r, s, t) \cdot B^{(m)}\right) t\right\|_{\ell_{p}} \\
& =\left(\left|\left(1-\left(\frac{\varepsilon}{2}\right)^{p}\right)^{\frac{1}{p}}-\left(1-\left(\frac{\varepsilon}{2}\right)^{p}\right)^{\frac{1}{p}}\right|^{p}+\left|\frac{\varepsilon}{2}-\left(-\frac{\varepsilon}{2}\right)\right|^{p}\right)^{\frac{1}{p}} \\
& =\varepsilon .
\end{aligned}
$$

To complete the estimate of the Gurarii on the modulus of convexity, it remains to calculate the infimum of $\|\alpha x+(1-\alpha) t\|_{\ell_{p}\left(r, s, t ; B^{(m)}\right)}^{p}$ for $0 \leq \alpha \leq 1$. We have

$$
\begin{aligned}
\inf _{0 \leq \alpha \leq 1}\|\alpha x+(1-\alpha) t\|_{\ell_{p}\left(r, s, t ; B^{(m)}\right)} & \\
= & \inf _{0 \leq \alpha \leq 1}\left\|\alpha\left(\bar{A}(r, s, t) \cdot B^{(m)}\right) x+(1-\alpha)\left(\bar{A}(r, s, t) \cdot B^{(m)}\right) t\right\|_{\ell_{p}} \\
= & \inf _{0 \leq \alpha \leq 1}\left[\left|\alpha\left(1-\left(\frac{\varepsilon}{2}\right)^{p}\right)^{\frac{1}{p}}+(1-\alpha)\left(1-\left(\frac{\varepsilon}{2}\right)^{p}\right)^{\frac{1}{p}}\right|^{p}\right. \\
& \left.+\left|\alpha\left(\frac{\varepsilon}{2}\right)+(1-\alpha)\left(-\frac{\varepsilon}{2}\right)\right|^{p}\right]^{\frac{1}{p}} \\
= & \inf _{0 \leq \alpha \leq 1}\left[1-\left(\frac{\varepsilon}{2}\right)^{p}+|2 \alpha-1|^{p}\left(\frac{\varepsilon}{2}\right)^{p}\right]^{\frac{1}{p}} \\
= & \left(1-\left(\frac{\varepsilon}{2}\right)^{p}\right)^{\frac{1}{p}} .
\end{aligned}
$$

Consequently, we get for $p \geq 1$ the inequality

$$
\beta_{l_{p}(u, v)}(\varepsilon) \leq 1-\left(1-\left(\frac{\varepsilon}{2}\right)^{p}\right)^{\frac{1}{p}}
$$

which is the desired result.

\section{Conclusion}

Recently the sequence space $X\left(r, s, t ; B^{(m)}\right)$ for $X \in\left\{\ell_{\infty}, c, c_{0}\right\}$ has been studied by Maji and Srivastava [36] which is obtained by combining the generalized means and the $m$ th order generalized difference operator $B^{(m)}(u, v)$. They characterized some compact operators on the spaces $X\left(r, s, t ; B^{(m)}\right)$ for $X \in\left\{\ell_{\infty}, c, c_{0}\right\}$ by using the Hausdorff measure of noncompactness. In this paper, we have derived some identities or estimates for the operator norms and the Hausdorff measures of noncompactness of certain matrix operators on the sequence space $\ell_{p}\left(r, s, t ; B^{(m)}\right)$. Further, by applying the Hausdorff measure of noncompactness, we obtained the necessary and sufficient conditions for such operators to be compact. In the last section, we have studied some geometric properties of this space, e.g. the property Banach-Saks type and Gurarii modulus of convexity. 
Authors' contributions

All authors of the manuscript have read and agreed to its content and are accountable for all aspects of the accuracy and integrity of the manuscript.

\section{Author details}

1 Department of Mathematics, Faculty of Science, King Abdulaziz University, P.O. Box 80203, Jeddah, 21589, Saudi Arabia.

2Department of Mathematics, Aligarh Muslim University, Aligarh, 202002, India.

\section{Acknowledgements}

The authors gratefully acknowledge the financial support from King Abdulaziz University, Jeddah, Saudi Arabia.

Received: 22 December 2015 Accepted: 11 July 2016 Published online: 04 August 2016

\section{References}

1. Wilansky, A: Summability through Functional Analysis. North-Holland Mathematics Studies, vol. 85. Elsevier, Amsterdam (1984)

2. Malkowsky, E, Rakočević, V: An introduction into the theory of sequence spaces and measures of noncompactness In: Zbornik Radova, vol. 9, pp. 143-234. Mat. Institut SANU, Beograd (2000)

3. Banaś, J, Goebel, K: Measures of Noncompactness in Banach Spaces. Lecture Notes in Pure and Appl. Math., vol. 60. Dekker, New York (1980)

4. Banaś, J, Mursaleen, M: Sequence Spaces and Measures of Noncompactness with Applications to Differential and Integral Equations. Springer, New Delhi (2014)

5. Goldenštein, LS, Gohberg, IT, Markus, AS: Investigations of some properties of bounded linear operators with their q-norms. Učen. Zap. Kishinevsk. Univ. 29, 29-36 (1957)

6. Abyar, E, Ghaemi, MB: Hausdorff measure of noncompactness of matrix operators on some sequence spaces of a double sequential band matrix. J. Inequal. Appl. 2015, 406 (2015)

7. Alotaibi, A, Mursaleen, M, Alamri, BAS, Mohiuddine, SA: Compact operators on some Fibonacci difference sequence spaces. J. Inequal. Appl. 2015, 203 (2015)

8. Başar, F, Malkowsky, E: The characterization of compact operators on spaces of strongly summable and bounded sequences. Appl. Math. Comput. 217, 5199-5207 (2011)

9. Başarir, M, Kara, EE: On some difference sequence spaces of weighted means and compact operators. Ann. Funct. Anal. 2(2), 116-131 (2011)

10. Başarir, M, Kara, EE: On compact operators on the Riesz $B^{(m)}$ difference sequence space. Iran. J. Sci. Technol. 35(A4), 279-285 (2011)

11. Başarir, M, Kara, EE: On the B-difference sequence space derived by generalized weighted mean and compact operators. J. Math. Anal. Appl. 391(1), 67-81 (2012)

12. Başarir, $M$, Kara, EE: On compact operators on the Riesz $B^{(m)}$ difference sequence spaces II. Iran. J. Sci. Technol. 36(A3), 371-376 (2012)

13. Başarir, M, Kara, EE: On the $m$ th order difference sequence space of generalized weighted mean and compact operators. Acta Math. Sci. 33(3), 797-813 (2013)

14. Clarkson, JA: Uniformly convex spaces. Trans. Am. Math. Soc. 40, 396-414 (1936)

15. Cui, YA, Hudzik, H: On the Banach-Saks and weak Banach-Saks properties of some Banach sequence spaces. Acta Sci. Math. 65, 179-187 (1999)

16. Day, MM: Uniform convexity in factor and conjugate spaces. Ann. Math. 45(2), 375-385 (1944)

17. Diestel, J: Sequences and Series in Banach Spaces. Graduate Texts in Math., vol. 92. Springer, Berlin (1984)

18. García-Falset, J: Stability and fixed points for nonexpansive mappings. Houst. J. Math. 20, 495-505 (1994)

19. García-Falset, J: The fixed point property in Banach spaces with NUS-property. J. Math. Anal. Appl. 215(2), 532-542 (1997)

20. Gurariĭ, Vl: On differential properties of the convexity moduli of Banach spaces. Mat. Issled. 2, 141-148 (1969)

21. Hudzik, H, Karakaya, V, Mursaleen, M, Şimşek, N: Banach-Saks type and GurariI modulus of convexity of some Banach sequence spaces. Abstr. Appl. Anal. 2014, Article ID 427382 (2014)

22. Knaust, H: Orlicz sequence spaces of Banach-Saks type. Arch. Math. 59, 562-565 (1992)

23. Kara, EE, Başarir, M: On some Euler $B^{(m)}$ difference sequence spaces and compact operators. J. Math. Anal. Appl. 379 499-511 (2011)

24. Kara, EE, Başarir, M, Konca, Ş: On some new weighted Euler sequence spaces and compact operators. Math. Inequal. Appl. 17(2), 649-664 (2014)

25. de Malafosse, B, Rakočević, V: Applications of measure of noncompactness in operators on the spaces $s_{\alpha}, s_{\alpha}^{0}, s_{\alpha}^{(c)}, \ell_{\alpha}^{p}$. J. Math. Anal. Appl. 323(1), 131-145 (2006)

26. Malkowsky, E: Characterization of compact operators between certain BK spaces. Filomat 27(3), 447-457 (2013)

27. Mursaleen, $M$, Karakaya, $V$, Polat, H, Simşek, N: Measure of noncompactness of matrix operators on some difference sequence spaces of weighted means. Comput. Math. Appl. 62, 814-820 (2011)

28. Mursaleen, M, Noman, AK: Compactness by the Hausdorff measure of noncompactness. Nonlinear Anal. 73(8), 2541-2557 (2010)

29. Mursaleen, M, Noman, AK: Hausdorff measure of noncompactness of certain matrix operators on the sequence spaces of generalized means. J. Math. Anal. Appl. 417, 96-111 (2014)

30. Aghajania, A, Allahyari, R, Mursaleen, M: A generalization of Darbo's theorem with application to the solvability of systems of integral equations. J. Comput. Appl. Math. 260, 68-77 (2014)

31. Banaś, J: Measures of noncompactness in the study of solutions of nonlinear differential and integral equations. Cent. Eur. J. Math. 10(6), 2003-2011 (2012)

32. Banaś, J, Lecko, M: Solvability of infinite systems of differential equations in Banach sequence spaces. J. Comput. Appl. Math. 137, 363-375 (2001)

33. Mursaleen, M, Mohiuddine, SA: Applications of measures of noncompactness to the infinite system of differential equations in $\ell_{p}$ spaces. Nonlinear Anal. 75, 2111-2115 (2012) 
34. Alotaibi, A, Mursaleen, M, Mohiuddine, SA: Application of measures of noncompactness to infinite system of linear equations in sequence spaces. Bull. Iran. Math. Soc. 41(2), 519-527 (2015)

35. Mursaleen, M, Noman, AK: On generalized means and some related sequence spaces. Comput. Math. Appl. 61(4), 988-999 (2011)

36. Maji, A, Srivastava, PD: On $B^{(m)}$-difference sequence spaces using generalized means and compact operators. Analysis 34(3), 257-281 (2014)

37. Maji, A, Srivastava, PD: Applications of the Hausdorff measure of noncompactness on the space $\ell_{p}\left(r, s, t ; B^{(m)}\right)$, $1 \leq p<\infty$. In: Mathematics and Computing 2013. Springer Proc. Math. Stat., vol. 91, pp. 271-281. Springer, New Delhi (2014)

Submit your manuscript to a SpringerOpen ${ }^{\circ}$ journal and benefit from:

- Convenient online submission

Rigorous peer review

- Immediate publication on acceptance

- Open access: articles freely available online

- High visibility within the field

- Retaining the copyright to your article

Submit your next manuscript at $\boldsymbol{s p r i n g e r o p e n . c o m ~}$ 